\title{
Clinical Study on the Correlation Between Blastocyst Morphological Score and Chromosome Euploidy
}

\author{
Min Pang, Jaynagul Hojabek, Qiong Zhen Zhao, Kun Zhang, Jian Sun and WeiDong Huang* \\ ${ }^{1}$ Reproductive medicine center, XinJiang JiaYin Hospital, China
}

Submission: May 15, 2020; Published: June 02, 2020

*Corresponding author: WeiDong Huang, Reproductive medicine center, Xinjiang JiaYin Hospital, 48 Hubei Road, Shuimogou District, Urumchi, Xinjiang Province, 830000, China

\begin{abstract}
Objective: To analyze the correlation between blastocyst morphological score and blastocyst chromosome abnormalities in Pre-implantation Genetic Screening (PGS) and Pre-implantation Genetic Diagnosis (PGD).

Methods: Patients were divided into the PGD group and the PGS group, the morphological scores of blastocysts and chromosome abnormalities were detected. The blastocysts morphological scores were classified as good, medium and poor levels. Chromosome detection results were divided into euploidy, aneuploidy and other abnormalities categories. SPSS software was applied to analyze the significant difference.

Results: PGS group detected 141 cases of blastocysts, and the euploidy rates of good, medium and poor blastocysts were 62.5\% (40/64), $58.1 \%(25 / 43)$ and $41.2 \%(14 / 34)$, respectively. The aneuploidy rates were $21.9 \%(14 / 64), 16.3 \%(7 / 43)$ and $47.1 \%(16 / 34)$, respectively. Other anomaly rates were $15.6 \%(10 / 64), 25.6 \%(11 / 43)$ and $11.8 \%(4 / 34)$, respectively. In the PGD group, the euploidy rates of 179 blastocysts were $44.1 \%$ (37/84), 40.0\% (20/50) and 40.0\% (18/45), respectively. The aneuploidy rates were $13.1 \%(11 / 84), 26.0 \%(13 / 50)$ and $35.6 \%$ (16/45), respectively. The other abnormal rates were $42.9 \%(36 / 84), 34.0 \%(17 / 50)$ and $24.4 \%(11 / 45)$.

Conclusion: In PGS group and PGD group, although the rate of chromosome abnormality increased with the decrease of blastocyst grade, there was no significant difference. There is a weak correlation between blastocyst morphology and aneuploidy. Among aneuploidy blastocysts, some obtained the highest morphological score, while some had poor morphological score. Therefore, morphological scores cannot be used to determine the blastocysts euploidy.
\end{abstract}

Keywords: Blastocyst; Morphological scoring; Chromosome aneuploidy; PGD; PGS

\section{Introduction}

In IVF-ET (In Vitro Fertilization-Embryo Transfer) treatment, the correct evaluation of embryonic development potential is the basic guarantee for a good pregnancy outcome [1]. Currently, most embryo assessment methods use morphological criteria, such as cell size, cell number, multinuclear, and percentage of cell debris in embryos [2,3]. However, there is little correlation between these characteristics and in vitro fertilization results, so morphological grading plays a limited role in guiding embryo viability. In most cases, the root causes of embryo block, implantation failure or spontaneous abortion are chromosome number abnormalities (aneuploidy). Aneuploidy has been found in human oocyte during in vitro fertilization as early as 30 years ago,[4] Many studies have confirmed that human oocytes and pre-implantation embryos are often affected by chromosomal abnormalities [5,6]. In fact, for women over 40 years old, more than half of the oocytes are aneuploidy. In most cases, chromosomal imbalance is fatal to embryos developing. Many studies are looking for the relationship between aneuploidy and morphological score in embryos $[7,8]$. Some relationships have been confirmed, for example, some aneuploidy embryos found cell division, uneven cell size, and the number of multinucleated or atypical cells. In most cases, however, they have a slightly correlations. There is no reliable morphological index of aneuploidy has been found in previous studies, which may be reasoned by technical constraints. Almost all studies and clinical investigations have assessed only a limited number of chromosomes in each embryo, so it is inevitable that some embryos classified as euploidy which are actually abnormal, 
thereby aneuploidy was not detected. In this study, we used nextgeneration sequencing (NGS), a molecular genetic method that can simultaneously detect all chromosome abnormalities [9]. Up to now, there are few reports on the relationship between blastocyst morphology and more detailed chromosomal abnormalities in blastocyst screening using NGS-like methods. Therefore, it is necessary to reassess the potential relationship between morphology and aneuploidy applying the data of whole chromosome screening.

\section{Methods and Materials}

\section{Samples}

From June 2017 to August 2019, 102 cases of pre-implantation genetic diagnosis were performed in our center. This study was approved by the Clinical Research Ethics Committee of the Xinjiang JiaYin hospital. All patients provided written informed consent before participation. They were divided into PGD group and PGS group. There were 53 carriers with abnormal chromosome structure. Among them, 10 cases were inverted, 24 cases were translocation, 4 case was derivation, 1 case was 47XYY, totally of 179 blastocysts were detected. There were 49 cases of pre-implantation genetic screening that have a total of 141 blastocysts. The main indications were age over 35 years old; recurrent abortion; children with chromosomal aneuploidy.

\section{Blastocyst score}

Blastocysts were scored by Gardner's scoring method. According to the expansion or hatching of blastocysts, blastocysts can be divided into 1 to 6 stages, and Morphological grading was performed according to the number of cells in the inner cell mass and trophoblastic layer. Internal cell grading according to the internal cell manifestation, it can be divided into three levels:

a. the number of cells is large and closely arranged.

b. the number of cells is small and loosely arranged.

c. the number of cells is very few or nonexistent.

Classification of trophoblastic layer: According to trophoblastic layer manifestations, it can be divided into three levels:

a. a large number of cells well-distributed around the cysts, closely arranged.

b. fewer cells loosely arranged.

c. very few cells or no cells.

Blastocysts are classified into three categories: good blastocysts ( $\geq 3 \mathrm{AA})$; medium blastocysts $(3,4,5,6, \mathrm{AB}$ and $\mathrm{BA}$; poor blastocyst $(3,4,5,6 \mathrm{BB}, \mathrm{AC}$ and $\mathrm{CA})$.

\section{Blastocyst biopsy}

Nutritional Ectoderm (TE) biopsy was performed on the 5th or 6th days, and immediately cryopreservation by vitrification after biopsy. 23 pairs of chromosomes were screened for aneuploidy by NGS analysis.

\section{Genetic detection}

After blastocyst biopsy, the obtained blastocyst trophoblast cells were sent to the genetic testing laboratory for genetic analysis. Pre-implantation chromosome aneuploidy detection kit (semiconductor sequencing) was used to detect chromosome aneuploidy, and on-line sequencing was carried out by Ion Proton high-throughput sequencing platform.

\section{Statistical analysis}

Data were processed and analyzed by SPSS17.0 statistical analysis software. The difference $\mathrm{p}<0.05$ was considered significant.

\section{Results}

\section{Blastocyst grading and chromosome detection results}

In the PGS group, 141 blastocysts were detected with good, medium and poor levels which with the euploidy rates of $62.5 \%$ (40/64), 58.1\% (25/43) and 41.2\% (14/34), respectively. The aneuploidy rates of $21.9 \%$ (14/64), 16.3\% (7/43) and $47.1 \%$ $(16 / 34)$ for good, medium and poor blastocysts. Other abnormal rates of $15.6 \%(10 / 64), 25.6 \%(11 / 43)$ and $11.8 \%(4 / 34)$, respectively. In the PGD group, the euploidy rates of 179 blastocysts were $44.1 \%$ (37/84), $40.0 \%$ (20/50) and $40.0 \%$ (18/45). The aneuploidy rates were $13.1 \%$ (11/84), 26.0\% (13/50) and $35.6 \%$ (16/45). The other abnormal rates were $42.9 \%$ (36/84), $34.0 \%$ $(17 / 50)$ and $24.4 \%(11 / 45)$, respectively.

The relationship between blastocyst grading and chromosomal abnormalities in both PGS and PGD groups, the rates of chromosomal abnormality increased with the decrease of blastocyst grade, but there was no significant difference. With the decrease of blastocyst grade, there was no significant difference in chromosome euploidy rate and other abnormal rate between PGS and PGD groups (Table 1). In good blastocysts, the euploidy rate of PGD group was lower than that of PGS group, but there was no significant difference; There was no significant difference in aneuploidy rates between PGD group and PGS group; Other abnormal rates of PGD group was higher than that of PGS group, and there was significant difference. This result accorded with the theory that chromosome abnormality carriers produced higher fragment abnormalities in gametes.

\section{Discussion}

The little correlation was found in blastocyst stage embryo morphology and euploidy rate (Table 2). Having a high scoring ICM and TE had no significant euploidy rate than those with poorer grades. Early studies reported the relationship between aneuploidy and suggest that the TE or ICM score was positively associated with the euploidy status of the embryo $[10,11]$. However, 
in most cases, the correlation is weak, and no morphological marker can accurately distinguish chromosomal abnormalities from aneuploidy embryos. It indicated that morphology could not guide the identification of embryonic aneuploidy. In addition, it may be related to unclear understanding of embryo classification as normal or aneuploidy, because previous studies used Fluorescence in Situ Hybridization (FISH) to assess only one fifth to half of the chromosomes of each cell. Later studies show that aneuploidy can affect any chromosome. Therefore, in early studies, some embryos inevitably misclassify undetected embryos with abnormal chromosomes. Besides, many data focus on the detection of individual cells to predict the effect of aneuploidy on the morphology of the whole embryo. The effect of chromosome microdeletion, microduplication abnormality was not considered. At the same time, embryos have high frequency of chromosome chimerism, occasionally diploid: aneuploid chimerism, so occur the probability of some misclassification of chromosome abnormalities in embryos. In this study, the relationship between blastocyst morphological score and chromosomal abnormalities was preliminarily analyzed because of the small amount of data, and the relationship between blastocyst morphological score and chromosomal abnormalities needs to be further studied through large data.

Table 1: Chromosome Detection Results of Different Blastocyst Levels in PGS and PGD Groups.

\begin{tabular}{|c|c|c|c|c|c|c|c|}
\hline \multirow{4}{*}{ PGD } & Grade & Euploidy & $P$ value & Aneuploidy & $P$ value & Other Abnormality & $P$ value \\
\hline & Good & $38.10 \%$ & \multirow{3}{*}{0.861} & $17.46 \%$ & \multirow{3}{*}{0.011} & $44.44 \%$ & \multirow{3}{*}{0.11} \\
\hline & Medium & $42.86 \%$ & & $20.00 \%$ & & $37.14 \%$ & \\
\hline & Poor & $37.93 \%$ & & $34.48 \%$ & & $27.59 \%$ & \\
\hline \multirow{3}{*}{ PGS } & Good & $65.85 \%$ & \multirow{3}{*}{0.122} & $21.95 \%$ & \multirow{3}{*}{0.005} & $12.20 \%$ & \multirow{3}{*}{0.242} \\
\hline & Medium & $52.00 \%$ & & $12.00 \%$ & & $36.00 \%$ & \\
\hline & Poor & $53.33 \%$ & & $40.00 \%$ & & $6.67 \%$ & \\
\hline
\end{tabular}

Table 2: The relationship between euploidy rates with age, indication, and morphology score.

\begin{tabular}{|c|c|c|c|c|}
\hline \multirow{2}{*}{\begin{tabular}{|c|} 
Variables \\
Ages(years)
\end{tabular}} & \multicolumn{2}{|c|}{ Euploidy rate } & \multicolumn{2}{|c|}{ P value } \\
\hline & PGD & PGS & PGD & PGS \\
\hline $35 \leq$ & $43.72 \%(73 / 167)$ & $45.45 \%(10 / 22)$ & \multirow{2}{*}{0.067} & \multirow{2}{*}{0.277} \\
\hline $35>$ & $16.66 \%(2 / 12)$ & $57.98 \%(69 / 119)$ & & \\
\hline \multicolumn{5}{|c|}{ Primary indications } \\
\hline $\mathrm{I}$ & $31.64 \%(25 / 79)$ & $60.41 \%(58 / 96)$ & \multirow{3}{*}{0.005} & \multirow{3}{*}{0.063} \\
\hline II & $36.11 \%(13 / 36)$ & $33.33 \%(5 / 15)$ & & \\
\hline III & $57.81 \%(37 / 64)$ & $43.33 \%(13 / 30)$ & & \\
\hline \multicolumn{5}{|l|}{ Morphology } \\
\hline Good & $44.05 \%(37 / 84)$ & $62.50 \%(40 / 64)$ & \multirow{3}{*}{0.861} & \multirow{3}{*}{0.122} \\
\hline Medium & $40.00 \%(20 / 50)$ & $58.14 \%(25 / 43)$ & & \\
\hline Poor & $40.00 \%(18 / 45)$ & $41.18 \%(14 / 34)$ & & \\
\hline \multicolumn{5}{|l|}{ ACM } \\
\hline $\mathrm{A}$ & $42.97 \%(52 / 121)$ & $61.85 \%(60 / 97)$ & \multirow{3}{*}{0.833} & \multirow{3}{*}{0.06} \\
\hline B & $38.88 \%(21 / 54)$ & $41.86 \%(18 / 43)$ & & \\
\hline $\mathrm{C}$ & $50.00 \%(2 / 4)$ & $100.00 \%(1 / 1)$ & & \\
\hline \multicolumn{5}{|l|}{$\mathrm{TE}$} \\
\hline $\mathrm{A}$ & $43.29 \%(42 / 97)$ & $60.52 \%(46 / 76)$ & \multirow{3}{*}{0.204} & \multirow{3}{*}{0.419} \\
\hline $\mathrm{B}$ & $38.75 \%(31 / 80)$ & $51.61 \%(32 / 62)$ & & \\
\hline $\mathrm{C}$ & $100.00 \%(2 / 2)$ & $33.33 \%(1 / 3)$ & & \\
\hline
\end{tabular}

I= translocation for PGD, recurrent abortion for PGS; II = inversion for PGD, advanced maternal age for PGS; III = derivatives for PGD, with chromosomal aneuploidy children for PGS; ACM = inner cell mass; TE = trophectoderm. Difference was considered significant when $P<0.05$. 


\section{References}

1. Gardner DK, Schoolcraft WB (1999) Culture and transfer of human blastocysts. Curr Opin Obstet Gynecol 11(3): 307-311.

2. Van RE, Mangelschots K, De ND, M Valkenburg, M Van de Meerssche, et al. (1999) Characterization of a top quality embryo, a step towards single-embryo transfer. Hum Reprod 14(9): 2345-2349.

3. Hardarson T, Hanson C, Sjögren A, K Lundin (2001) Human embryos with unevenly sized blastomeres have lower pregnancy and implantation rates: indications for aneuploidy and multinucleation. Hum reprod 16(2): 313-318.

4. RR Angell, R J Aitken, PF van Look, MA Lumsden, AA Templeton (1983) Chromosome abnormalities in human embryos after in vitro fertilization. Nature 303(5915): 336-338.

5. Fragouli E, Katz-Jaffe M, Alfarawati S, John Stevens, Pere Colls, et al. (2010) Comprehensive chromosome screening of polar bodies and blastocysts from couples experiencing repeated implantation failure. Fertility \& Sterility 94(3): 875-887.

6. Kohn TP, Kohn JR, Darilek S, Ranjith Ramasamy, Larry Lipshultz, et al. (2016) Genetic counseling for men with recurrent pregnancy loss or recurrent implantation failure due to abnormal sperm chromosomal aneuploidy. J Assist Reprod Genet 33(5): 571-576.
7. Magli MC, Gianaroli L, Ferraretti AP, Alessandra Ruberti, Michela Lappi, et al. (2007) Embryo morphology and development are dependent on the chromosomal complement. Fertility \& Sterility 87(3): 534-541.

8. Majumdar G, Majumdar A, Verma IC, Upadhyaya KC (2017) Relationship Between Morphology, Euploidy and Implantation Potential of Cleavage and Blastocyst Stage Embryos. Journal of human reproductive sciences 10(1): 49-57.

9. Fiorentino F, Anil Biricik, Sara Bono, Letizia Spizzichino, Ettore Cotroneo, et al. (2014) Development and validation of a next-generation sequencing-based protocol for 24-chromosome aneuploidy screening of embryos. Fertility and Sterility 101(5): 1375-1382.

10. Munné S, Chen S, Colls P, J Garrisi, X Zheng, et al. (2007) Maternal age morphology, development and chromosome abnormalities in over 6000 cleavage-stage embryos. Reprod Biomed Online 14(5): 628-634.

11. Munné S, Alikani M, Tomkin G, Grifo J (1995) Cohen J Embryo morphology, developmental rates, and maternal age are correlated with chromosome abnormalities. Fertil Steril 64(2): 382-391.

Your next submission with Juniper Publishers will reach you the below assets

- Quality Editorial service

- Swift Peer Review

- Reprints availability

- E-prints Service

- Manuscript Podcast for convenient understanding

- Global attainment for your research

- Manuscript accessibility in different formats

( Pdf, E-pub, Full Tsext, Audio)

- Unceasing customer service

Track the below URL for one-step submission

https://juniperpublishers.com/online-submission.php 